\title{
Formulation of predictive model for the compressive strength of oyster shell powder-cement concrete using Scheffe's simplex lattice theory
}

\author{
ОвіEKWE A. UBACHUKWU - Department of Civil Engineering, \\ Michael Okpara University of Agriculture, Nigeria - obiubachukwu@yahoo.com \\ FIDELIS O. OKAFOR - Department of Civil Engineering, \\ University of Nigeria, Nigeria - fidelis.okafor@unn.edu.ng \\ Érkezett: 2020. 03. 24. " Received: 24. 03. 2020. " https://doi.org/10.14382/epitoanyag-jsbcm.2020.34
}

\begin{abstract}
This empirical study was carried out to formulate and validate a predictive model for the compressive strength of oyster shell powder-cement concrete using Scheffe's simplex lattice theory, so as to ensure economic usage of readily available oyster shells. A total of 90 cubes of concrete were cast to formulate and validate the model for the compressive strength of oyster shell powder (OSP)-cement concrete using Scheffe's $(5,2)$ simplex lattice theory. The formulated model was tested for adequacy using the Student t-test. It was observed that the model results agree with those of the experiments. Hence, the model is adequate and can predict the compressive strength, given the mix proportions. The model gave highest compressive strength of $30.81 \mathrm{~N} / \mathrm{mm}^{2}$ corresponding to mix ratio of 0.54:0.815:2.045:3.925:0.185 for water, cement, sand, granite and OSP respectively. The developed model also gave minimum compressive strength of $17.85 \mathrm{~N} / \mathrm{mm}^{2}$ corresponding to mix ratio of 0.525:0.825:2.2:4.05:0.175 for water, cement, sand, granite and OSP respectively. With this formulated model, any point on the simplex can easily be derived.

Key words: concrete, oyster shells, compressive strength, Scheffe, simplex

Kulcsszavak: beton, osztriga héj, nyomószilárdság, Scheffe, szimplex
\end{abstract}

\section{Introduction}

When a product is formed by mixing together two or more ingredients, the product is called a mixture and the ingredients are called mixture components [1]. In the case of concrete (mixture) and mixture components (cement, sand, granite, water and admixture or supplementary cementitious material), there is need to develop a way of optimally combining these ingredients, with a view to economizing our scarce resources, without compromise on the rheological and hardened properties of concrete produced. According to [2], it is important to find the optimum dosage and substitution ratio, because application of supplementary cementing materials over the optimum amount may reduce the performance, both in strength and durability parameters. One of the purposes of a mixture experiment is to find the best proportion of each component and the best value of each process variable, in order to optimize a single response or multiple responses simultaneously. A comprehensive methodology for mixture experiment was first proposed by $[3,4]$. Scheffe introduced the $\{\mathrm{q}, \mathrm{m}\}$ simplex lattice design and simplex centroid designs. If the number of components is not large and a high order polynomial is needed in order to accurately describe the response surface; then, a simplex lattice design can be used [5]. Scheffe's model is most times referred to as mixture model. They differ from the usual regression model due to correlation among all components in the mixture designs. Another difference is that the intercept term in the model is not usually included in the regression model [6]. Scheffe expressed the functional relationship between the investigated property and mixture components. Scheffe's ideas endure as primary recourse for practitioners of mixture experiments [7]. In a bid to reduce air and water pollutions, global warming, cost of construction and environmental nuisance, some researchers have used oyster shell powder as supplementary cementitious material to produce ecologically and economically friendly concrete [8-13]. The use of supplementary cementing materials in concrete may help in reducing the large carbon dioxide emission that result from production of Portland cement [14]. However, none of those researchers was able to come up with a model to optimize these mixture components as a predictor of compressive strength, given the mix proportion and vice versa. Hence, the present study will focus on model formulation and validation of oyster shell powder-cement concrete using the Scheffe's simplex lattice theory.

\section{Materials and methods}

\subsection{Materials}

Dangote brand of ordinary Portland cement was used in this research and it conformed to the requirements of [15]. The sand was sourced from Imo River in Imo State. It was sieved through $10 \mathrm{~mm}$ British standards test sieve to remove cobbles. The sand was sharp and free from deleterious substances and conforms to the requirements of [16]. The granite was sourced from the quarry site at Ishiagu, Ebonyi State, Nigeria. The maximum size of aggregate used for this work is $20 \mathrm{~mm}$ diameter. It was thoroughly flushed with water to reduce the level of impurities 
and organic matter that might have intruded during quarrying; to conform to requirements [17]. The water used for the study was obtained from borehole. The water was clean and free from any visible impurities. It conformed to the requirements of [18]. The water does not contain harmful constituents in such quantities as may be detrimental to the setting, hardening and durability of the concrete. Oyster Shell Powder was obtained from oyster shells littered at Okwagwe River, Delta State, after washing, sun drying, crushing and sieving with $150 \mu \mathrm{m}$ sieve. A total of 90 cubes of concrete were cast and cured for 28 days. 15 runs with three replicates each for the model compressive strength and 15 runs with three replicates each for validation of the model (control).

\subsection{Method}

\subsubsection{Design of experiment}

The OSP-cement concrete is made up of five components: water, cement, sand, granite and OSP which we can designate as $X_{1}, X_{2}, X_{3}, X_{4}$ an $d_{X_{5}}$ respectively. Where, $X_{1}$ represents the volume fraction of component. The volume fractions of the components sum to one, and the region defined by this constraint is the regular tetrahedron (or simplex) shown in Fig. 1.

Each vertex of the tetrahedron represents the pure component. For example, the vertex labelled $\mathrm{X}_{1}$ is the pure water mixture with $\mathrm{X}_{1}=1, \mathrm{X}_{2}=0, \mathrm{X}_{3}=0, \mathrm{X}_{4}=0$ and $\mathrm{X}_{5}=0$ or $(1,0,0,0,0)$.

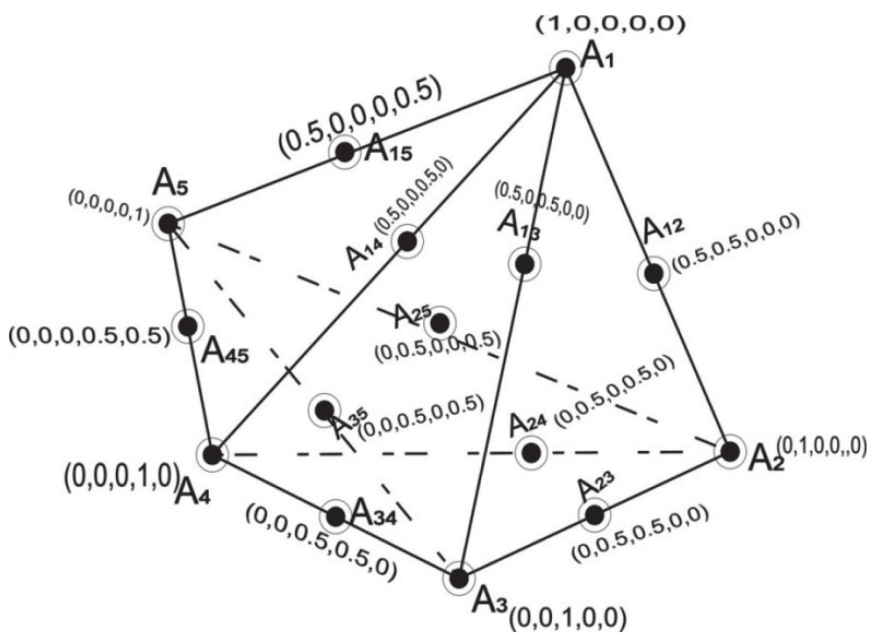

Fig. 1. A $(5,2)$ Scheffe's simplex lattice with 15 experimental runs

1. ábra $(5,2)$ méretü Scheffe szimplex rács 15 vizsgálati ponttal

All responses (properties) of interest would be measured for each mixture in the design and modelled as a function of the components. Here, polynomial functions will be used. The number of coefficients, $n$, of the polynomial is determined using equation

$n=\frac{(q+m-1) !}{(q-1) ! * m !}$

Where, $q$ is the number of components of the mixture, and $\mathrm{m}$ is the degree of the polynomial. Thus, for $q=5$ and $m=2$ as in second degree polynomial, $n=15$ signifying that for a $(q, m)=(5,2)$ simplex design, we have 15 coefficients of the polynomial function; thus, 15 experimental runs.
The mixture constraint, according to [3] implies that $0 \leq \mathrm{X}_{\mathrm{i}} \leq 1$, for $\mathrm{i}=1,2, \ldots, \mathrm{q}$

For a five - component mixture,

$\mathrm{X}_{1}+\mathrm{X}_{2}+\mathrm{X}_{3}+\mathrm{X}_{4}+\mathrm{X}_{5}=1$ (3)

The linear polynomial model for a response $\mathrm{y}$ is $\mathrm{Y}=\mathrm{b}^{*}+\mathrm{b}^{*} \mathrm{X}_{1}+\mathrm{b}_{2}^{*} \mathrm{X}_{2}+\mathrm{b}_{3}^{*} \mathrm{X}_{3}+\mathrm{b}^{*} \mathrm{X}_{4}+\mathrm{b}_{5}^{*} \mathrm{X}_{5}+\mathrm{e}$

Where $b^{*}$ are constants and $e$, the random error term, represents the combined effects of all variables not included in the model. The form of Eq. (4) is called the Scheffe's linear mixture polynomial. If there is curvature in the system, a polynomial of higher degree such as the second order model should be sought. Hence, the quadratic model is given by Eq. (5).

$\mathrm{Y}=\mathrm{b}_{0}^{*}+\mathrm{b}_{1}^{*} \mathrm{X}_{1}+\mathrm{b}_{2}^{*} \mathrm{X}_{2}+\mathrm{b}_{3}^{*} \mathrm{X}_{3}+\mathrm{b}_{4}^{*} \mathrm{X}_{4}+\mathrm{b}_{5}^{*} \mathrm{X}_{5}+\mathrm{b}_{12}^{*} \mathrm{X}_{1} \mathrm{X}_{2}+$ $\mathrm{b}_{13}^{*} \mathrm{X}_{1} \mathrm{X}_{3}+\mathrm{b}^{*}{ }_{14} \mathrm{X}_{1} \mathrm{X}_{4}+\mathrm{b}^{*}{ }_{15} \mathrm{X}_{1} \mathrm{X}_{5}+\mathrm{b}^{*}{ }_{23} \mathrm{X}_{2} \mathrm{X}_{3}+\mathrm{b}^{*}{ }_{24} \mathrm{X}_{2} \mathrm{X}_{4}$ $+\mathrm{b}_{25}^{*} \mathrm{X}_{2} \mathrm{X}_{5}+\mathrm{b}_{34}^{*} \mathrm{X}_{3} \mathrm{X}_{4}+\mathrm{b}^{*}{ }_{35} \mathrm{X}_{3} \mathrm{X}_{5}+\mathrm{b}_{45}^{*} \mathrm{X}_{4} \mathrm{X}_{5}+\mathrm{b}^{*}{ }_{11} \mathrm{X}_{1}^{2}+$ $\mathrm{b}_{22}^{*} \mathrm{X}_{2}^{2}+\mathrm{b}_{33}^{*} \mathrm{X}_{3}^{2}+\mathrm{b}_{44}^{*} \mathrm{X}_{4}{ }^{2}+\mathrm{b}_{55}^{*} \mathrm{X}_{5}^{2}+\mathrm{e}$

The challenge which any model developed using polynomial in Eq. (4) is that the developed model will always give an expected response, even when all the components are absent (zero). This limitation is due to the presence of $b_{o}$ and $e$, random error in the polynomials [19]. Scheffe's model of Eq. (5) overcomes this weakness. Transformation of Eq. (5) gives Eq. (6) below

$\widehat{Y}=\beta_{1} X_{1}+\beta_{2} X_{2}+\beta_{3} X_{3}+\beta_{4} X_{4}+\beta_{5} X_{5}+\beta_{12} X_{1} X_{2}+\beta_{13} X_{1} X_{3}+$ $\beta_{14} X_{1} X_{4}+\beta_{15} X_{1} X_{5}+\beta_{23} X_{2} X_{3}+\beta_{24} X_{2} X_{4}+\beta_{25} X_{2} X_{5}+\beta_{34} X_{3} X_{4}+$ $\beta_{35} X_{3} X_{5}+\beta_{45} X_{4} X_{5}$

Eq. (6) is the regression equation for the $(5,2)$ Scheffe's second degree canonical polynomial. The determination of the values of the coefficients in Eq. (6) will complete the model equation. Where:

$\mathrm{Y}_{1}=\beta_{1}, \mathrm{Y}_{2}=\beta_{2}, \mathrm{Y}_{3}=\beta_{3}, \mathrm{Y}_{4}=\beta_{4}, \mathrm{Y}_{5}=\beta_{5}$,

$\beta_{12}=4 \mathrm{Y}_{12}-2 \mathrm{Y}_{1}-2 \mathrm{Y}_{2}, \beta_{13}=4 \mathrm{Y}_{13}-2 \mathrm{Y}_{1}-2 \mathrm{Y}_{3}$,

$\beta_{14}=4 Y_{14}-2 Y_{1}-2 Y_{4}, \beta_{15}=4 Y_{15}-2 Y_{1}-2 Y_{5}$,

$\beta_{23}=4 \mathrm{Y}_{23}-2 \mathrm{Y}_{2}-2 \mathrm{Y}_{3}, \beta_{24}=4 \mathrm{Y}_{24}-2 \mathrm{Y}_{2}-2 \mathrm{Y}_{4}$,

$\beta_{25}=4 Y_{25}-2 Y_{2}-2 Y_{5}, \beta_{34}=4 Y_{34}-2 Y_{3}-2 Y_{4}$,

$\beta_{35}=4 \mathrm{Y}_{35}-2 \mathrm{Y}_{3}-2 \mathrm{Y}_{5} \beta_{45}=4 \mathrm{Y}_{45}-2 \mathrm{Y}_{4}-2 \mathrm{Y}_{5}$

\subsubsection{Concrete mix ratios for the formulation of regression model}

According to [20], the relationship between the actual and the pseudo mix ratios are given by

$\{Z\}=[A]\{X\}$

$\{\mathrm{X}\}=\left[\mathrm{A}^{-1}\right]\{\mathrm{Z}\}$

Where $Z, A$, and $X$ are respectively the real mix ratios, coefficient of relation matrix and pseudo mix ratios. The value of matrix A will be obtained from the first five real mix ratios. The first five mix ratios are: $Z_{1}[0.45: 0.95: 2.00: 4.00: 0.05]$, $Z_{2}[0.48: 0.90: 1.85: 3.75: 0.10], \quad Z_{3}[0.5: 0.85: 2.15: 4.15: 0.15]$, $Z_{4}[0.55: 0.80: 2.25: 3.95: 0.20], Z_{5}[0.60: 0.75: 1.75: 3.65: 0.25]$ and the corresponding pseudo mix ratios at the vertices of the tetrahedron (simplex) are $\mathrm{X}_{1}[1: 0: 0: 0: 0], \mathrm{X}_{2}$ [0:1:0:0:0], $\mathrm{X}_{3}[0: 0: 1: 0: 0], \mathrm{X}_{4}[0: 0: 0: 1: 0], \mathrm{X}_{5}[0: 0: 0: 0: 0: 1]$ as shown in Fig. 1.

This quadratic model involves 15 parameters, so design will be at least 15 points in order to fit the model. However, multiple points or complete replicates are required to provide sufficient degrees of freedom to test the adequacy of the fit. 
Matrix $A$ is the transpose of the first five mix ratios and these are as shown below:

$[A]=\left|\begin{array}{ccccc}0.45 & 0.48 & 0.5 & 0.55 & 0.60 \\ 0.95 & 0.90 & 0.85 & 0.80 & 0.75 \\ 2.00 & 1.85 & 2.15 & 2.25 & 1.75 \\ 4.00 & 3.75 & 4.15 & 3.95 & 3.65 \\ 0.05 & 0.10 & 0.15 & 0.20 & 0.25\end{array}\right|$

With the substitution of the other pseudo mix ratios at the midpoint of the simplex into Eq. (8), we obtain the 10 remaining real mix ratios. Hence, the mix ratios, both real and pseudo at the vertices and midpoints of the tetrahedron are as given in Table 1.
In order to statistically test the validity of the regression model for the compressive strength of OSP-cement concrete, fifteen additional mixes (control) were made as given in Table 2 .

The results obtained from the 28-compressive strength for model formulation were fitted into the regression equation to form the regression model. The model was validated using the results of 28-day compressive strength obtained using the control mix ratios.

\begin{tabular}{|c|c|c|c|c|c|c|c|c|c|c|}
\hline \multirow{2}{*}{ Points } & \multicolumn{5}{|c|}{ Real mix ratios } & \multicolumn{5}{|c|}{ Pseudo mix ratios } \\
\hline & $\begin{array}{c}\text { Water } \\
\mathrm{Z}_{1}\end{array}$ & $\begin{array}{c}\text { Cement } \\
\mathrm{Z}_{2}\end{array}$ & $\begin{array}{c}\text { Sand } \\
\mathrm{Z}_{3}\end{array}$ & $\begin{array}{c}\text { Granite } \\
Z_{4}\end{array}$ & $\begin{array}{c}\text { OSP } \\
Z_{5}\end{array}$ & $\begin{array}{c}\text { Water } \\
\mathrm{X}_{1}\end{array}$ & $\begin{array}{c}\text { Cement } \\
\mathrm{X}_{2}\end{array}$ & $\begin{array}{c}\text { Sand } \\
\mathrm{X}_{3}\end{array}$ & $\underset{X_{4}}{\text { Granite }}$ & $\begin{array}{c}\text { OSP } \\
X_{5}\end{array}$ \\
\hline$Y_{1}$ & 0.45 & 0.95 & 2.00 & 4.00 & 0.05 & 1.0 & 0.0 & 0.0 & 0.0 & 0.0 \\
\hline $\mathbf{Y}_{2}$ & 0.48 & 0.90 & 1.85 & 3.75 & 0.10 & 0.0 & 1.0 & 0.0 & 0.0 & 0.0 \\
\hline$Y_{3}$ & 0.50 & 0.85 & 2.15 & 4.15 & 0.15 & 0.0 & 0.0 & 1.0 & 0.0 & 0.0 \\
\hline$Y_{4}$ & 0.55 & 0.8 & 2.25 & 3.95 & 0.20 & 0.0 & 0.0 & 0.0 & 1.0 & 0.0 \\
\hline$Y_{5}$ & 0.60 & 0.75 & 1.75 & 3.65 & 0.25 & 0.0 & 0.0 & 0.0 & 0.0 & 1.0 \\
\hline$Y_{12}$ & 0.465 & 0.925 & 1.925 & 3.875 & 0.075 & 0.5 & 0.5 & 0.0 & 0.0 & 0.0 \\
\hline$Y_{13}$ & 0.475 & 0.90 & 2.075 & 4.075 & 0.100 & 0.5 & 0.0 & 0.5 & 0.0 & 0.0 \\
\hline$Y_{14}$ & 0.500 & 0.875 & 2.125 & 3.975 & 0.125 & 0.5 & 0.0 & 0.0 & 0.5 & 0.0 \\
\hline$Y_{15}$ & 0.525 & 0.850 & 1.875 & 3.825 & 0.150 & 0.5 & 0.0 & 0.0 & 0.0 & 0.5 \\
\hline$Y_{23}$ & 0.490 & 0.875 & 2.000 & 3.950 & 0.125 & 0.0 & 0.5 & 0.5 & 0.0 & 0.0 \\
\hline$Y_{24}$ & 0.515 & 0.850 & 2.050 & 3.850 & 0.150 & 0.0 & 0.5 & 0.0 & 0.5 & 0.0 \\
\hline$Y_{25}$ & 0.540 & 0.825 & 1.800 & 3.700 & 0.175 & 0.0 & 0.5 & 0.0 & 0.0 & 0.5 \\
\hline$Y_{34}$ & 0.525 & 0.825 & 2.200 & 4.050 & 0.175 & 0.0 & 0.0 & 0.5 & 0.5 & 0.0 \\
\hline $\mathbf{Y}_{35}$ & 0.550 & 0.800 & 1.950 & 3.900 & 0.200 & 0.0 & 0.0 & 0.5 & 0.0 & 0.5 \\
\hline $\mathbf{Y}_{45}$ & 0.575 & 0.775 & 2.000 & 3.800 & 0.225 & 0.0 & 0.0 & 0.0 & 0.5 & 0.5 \\
\hline
\end{tabular}

Table 1 Concrete mix ratios for model formulation

1. táblázat A modellalkotáshoz használt beton összetétel arányok

\begin{tabular}{|c|c|c|c|c|c|c|c|c|c|c|}
\hline \multirow[b]{2}{*}{ Point } & \multicolumn{5}{|c|}{ Real mix ratios } & \multicolumn{5}{|c|}{ Pseudo mix ratios } \\
\hline & $\begin{array}{c}\text { Water } \\
\mathrm{Z}_{1}\end{array}$ & $\begin{array}{c}\text { Cement } \\
\mathrm{Z}_{2}\end{array}$ & $\begin{array}{c}\text { Sand } \\
\mathbf{Z}_{3}\end{array}$ & $\begin{array}{c}\text { Granite } \\
\mathrm{Z}_{4}\end{array}$ & $\begin{array}{c}\text { OSP } \\
Z_{5}\end{array}$ & $\begin{array}{c}\text { Water } \\
\mathbf{X}_{1}\end{array}$ & $\begin{array}{c}\text { Cement } \\
\mathrm{X}_{2}\end{array}$ & $\begin{array}{c}\text { Sand } \\
\mathrm{X}_{3}\end{array}$ & $\begin{array}{c}\text { Granite } \\
\mathrm{X}_{4}\end{array}$ & $\begin{array}{c}\text { OSP } \\
X_{5}\end{array}$ \\
\hline$C_{1}$ & 0.495 & 0.875 & 2.0625 & 3.9625 & 0.1250 & 0.25 & 0.25 & 0.25 & 0.25 & 0.0 \\
\hline $\mathrm{C}_{2}$ & 0.5075 & 0.8625 & 1.9375 & 3.8875 & 0.1375 & 0.25 & 0.25 & 0.25 & 0.0 & 0.25 \\
\hline $\mathrm{C}_{3}$ & 0.5200 & 0.8500 & 1.9625 & 3.8375 & 0.1500 & 0.25 & 0.25 & 0.0 & 0.25 & 0.25 \\
\hline $\mathrm{C}_{4}$ & 0.525 & 0.8375 & 2.0375 & 3.9375 & 0.1625 & 0.25 & 0.0 & 0.25 & 0.25 & 0.25 \\
\hline$C_{5}$ & 0.5325 & 0.8250 & 2.0000 & 3.8750 & 0.1750 & 0.0 & 0.25 & 0.25 & 0.25 & 0.25 \\
\hline$C_{6}$ & 0.5160 & 0.8500 & 2.000 & 3.9000 & 0.1500 & 0.2 & 0.2 & 0.2 & 0.2 & 0.2 \\
\hline$C_{7}$ & 0.4840 & 0.8900 & 2.0250 & 3.9650 & 0.1100 & 0.3 & 0.3 & 0.3 & 0.1 & 0.0 \\
\hline $\mathrm{C}_{8}$ & 0.4890 & 0.8850 & 1.9750 & 3.9350 & 0.1150 & 0.3 & 0.3 & 0.3 & 0.0 & 0.1 \\
\hline $\mathrm{C}_{9}$ & 0.5040 & 0.8700 & 2.0050 & 3.8750 & 0.1300 & 0.3 & 0.3 & 0.0 & 0.3 & 0.1 \\
\hline$C_{10}$ & 0.5100 & 0.8550 & 2.0950 & 3.9950 & 0.1450 & 0.3 & 0.0 & 0.3 & 0.3 & 0.1 \\
\hline$C_{11}$ & 0.5190 & 0.8400 & 2.0500 & 3.9200 & 0.1600 & 0.0 & 0.3 & 0.3 & 0.3 & 0.1 \\
\hline$C_{12}$ & 0.5400 & 0.8150 & 2.0450 & 3.9250 & 0.1850 & 0.1 & 0.0 & 0.3 & 0.3 & 0.3 \\
\hline$C_{13}$ & 0.534 & 0.8300 & 1.9550 & 3.8050 & 0.1700 & 0.1 & 0.3 & 0.0 & 0.3 & 0.3 \\
\hline$C_{14}$ & 0.519 & 0.8450 & 1.9250 & 3.8650 & 0.1550 & 0.1 & 0.3 & 0.3 & 0.0 & 0.3 \\
\hline$C_{15}$ & 0.504 & 0.8600 & 2.0750 & 3.9550 & 0.1400 & 0.1 & 0.3 & 0.3 & 0.3 & 0.0 \\
\hline
\end{tabular}

Table 2 Concrete mix ratios (control) for model validation

2. táblázat A modell validálásához használt beton összetétel arányok 


\begin{tabular}{|c|c|c|c|c|c|c|}
\hline Response & Replicate & $\begin{array}{l}\text { Average mass } \\
\text { (kg) }\end{array}$ & Volume $\left(\mathrm{m}^{3}\right)$ & Crushing load (N) & $\begin{array}{c}\text { Compressive } \\
\text { strength }\left(\mathbf{N} / \mathbf{m m}^{2}\right)\end{array}$ & $\begin{array}{l}\text { Average compressive } \\
\text { strength }\left(\mathrm{N} / \mathrm{mm}^{2}\right)\end{array}$ \\
\hline Y1 & A & 8.20 & 0.003375 & 400000 & 17.78 & 19.48 \\
\hline Y1 & B & & & 490000 & 21.78 & \\
\hline Y1 & C & & & 425000 & 18.89 & \\
\hline Y2 & A & 8.72 & 0.003375 & 590000 & 26.22 & 29.01 \\
\hline Y2 & B & & & 730000 & 32.44 & \\
\hline Y2 & C & & & 638000 & 28.36 & \\
\hline Y3 & A & 8.28 & 0.003375 & 450000 & 20.00 & 19.56 \\
\hline Y3 & B & & & 430000 & 19.11 & \\
\hline Y3 & C & & & 440000 & 19.56 & \\
\hline Y4 & A & 8.38 & 0.003375 & 500000 & 22.22 & 21.93 \\
\hline Y4 & B & & & 460000 & 20.44 & \\
\hline Y4 & C & & & 520000 & 23.11 & \\
\hline Y5 & A & 8.33 & 0.003375 & 400000 & 17.78 & 19.21 \\
\hline Y5 & B & & & 482000 & 21.42 & \\
\hline Y5 & C & & & 415000 & 18.44 & \\
\hline Y12 & A & 8.48 & 0.003375 & 650000 & 28.89 & 30.81 \\
\hline $\mathrm{Y} 12$ & B & & & 730000 & 32.44 & \\
\hline $\mathrm{Y} 12$ & C & & & 700000 & 31.11 & \\
\hline Y13 & A & 8.68 & 0.003375 & 700000 & 31.11 & 27.41 \\
\hline Y13 & B & & & 550000 & 24.44 & \\
\hline Y13 & C & & & 600000 & 26.67 & \\
\hline Y14 & A & 8.77 & 0.003375 & 670000 & 29.78 & 25.93 \\
\hline Y14 & B & & & 530000 & 23.56 & \\
\hline Y14 & C & & & 550000 & 24.44 & \\
\hline Y15 & A & 8.70 & 0.003375 & 580000 & 25.78 & 24.74 \\
\hline Y15 & B & & & 570000 & 25.33 & \\
\hline Y15 & C & & & 520000 & 23.11 & \\
\hline Y23 & A & 8.77 & 0.003375 & 505000 & 22.44 & 23.48 \\
\hline Y23 & B & & & 535000 & 23.78 & \\
\hline Y23 & C & & & 545000 & 24.22 & \\
\hline Y24 & A & 8.77 & 0.003375 & 615000 & 27.33 & 26.59 \\
\hline Y24 & B & & & 585000 & 26.00 & \\
\hline Y24 & C & & & 595000 & 26.44 & \\
\hline Y25 & A & 8.63 & 0.003375 & 540000 & 24.00 & 23.70 \\
\hline Y25 & B & & & 510000 & 22.67 & \\
\hline Y25 & C & & & 550000 & 24.44 & \\
\hline Y34 & A & 8.18 & 0.003375 & 455000 & 20.22 & 17.85 \\
\hline Y34 & B & & & 385000 & 17.11 & \\
\hline Y34 & $C$ & & & 365000 & 16.22 & \\
\hline Y35 & A & 8.15 & 0.003375 & 440000 & 19.56 & 18.89 \\
\hline Y35 & B & & & 420000 & 18.67 & \\
\hline Y35 & C & & & 415000 & 18.44 & \\
\hline Y45 & A & 8.42 & 0.003375 & 425000 & 18.89 & 18.30 \\
\hline Y45 & B & & & 402000 & 17.87 & \\
\hline Y45 & $C$ & & & 408000 & 18.13 & \\
\hline
\end{tabular}




\begin{tabular}{|c|c|c|c|c|c|c|}
\hline $\begin{array}{l}\text { Response } \\
\text { (control) }\end{array}$ & Replicate & Average weight (kg) & Volume $\left(\mathrm{m}^{3}\right)$ & Crushing load (N) & $\begin{array}{c}\text { Compressive } \\
\text { strength }\left(\mathrm{N} / \mathrm{mm}^{2}\right)\end{array}$ & $\begin{array}{c}\text { Average compressive } \\
\text { strength }\left(\mathrm{N} / \mathrm{mm}^{2}\right)\end{array}$ \\
\hline $\mathrm{C} 1$ & A & 8.52 & 0.003375 & 480000 & 21.33 & 21.85 \\
\hline $\mathrm{C} 1$ & $B$ & & & 500000 & 22.22 & \\
\hline C1 & $\mathrm{C}$ & & & 495000 & 22.00 & \\
\hline $\mathrm{C} 2$ & $A$ & 7.45 & 0.003375 & 640000 & 28.44 & 24.59 \\
\hline $\mathrm{C} 2$ & $B$ & & & 510000 & 22.67 & \\
\hline $\mathrm{C} 2$ & $\mathrm{C}$ & & & 510000 & 22.67 & \\
\hline C3 & A & 8.45 & 0.003375 & 540000 & 24.00 & 22.59 \\
\hline C3 & $B$ & & & 470000 & 20.89 & \\
\hline C3 & $\mathrm{C}$ & & & 515000 & 22.89 & \\
\hline $\mathrm{C} 4$ & A & 8.68 & 0.003375 & 610000 & 27.11 & 24.59 \\
\hline C4 & $B$ & & & 525000 & 23.33 & \\
\hline $\mathrm{C} 4$ & $\mathrm{C}$ & & & 525000 & 23.33 & \\
\hline $\mathrm{C} 5$ & $A$ & 8.65 & 0.003375 & 550000 & 24.44 & 24.44 \\
\hline $\mathrm{C} 5$ & $B$ & & & 580000 & 25.78 & \\
\hline $\mathrm{C} 5$ & $\mathrm{C}$ & & & 520000 & 23.11 & \\
\hline C12 & $A$ & 8.33 & 0.003375 & 540000 & 24.00 & 25.26 \\
\hline $\mathrm{C} 12$ & $B$ & & & 580000 & 25.78 & \\
\hline $\mathrm{C} 12$ & $\mathrm{C}$ & & & 585000 & 26.00 & \\
\hline C13 & $A$ & 8.20 & 0.003375 & 575000 & 25.56 & 25.93 \\
\hline C13 & $B$ & & & 580000 & 25.78 & \\
\hline $\mathrm{C} 13$ & $\mathrm{C}$ & & & 595000 & 26.44 & \\
\hline C14 & A & 8.57 & 0.003375 & 570000 & 25.33 & 26.67 \\
\hline C14 & $B$ & & & 620000 & 27.56 & \\
\hline C14 & $\mathrm{C}$ & & & 610000 & 27.11 & \\
\hline C15 & $A$ & 8.47 & 0.003375 & 560000 & 24.89 & 24.79 \\
\hline C15 & $B$ & & & 595000 & 26.44 & \\
\hline C15 & $\mathrm{C}$ & & & 518000 & 23.02 & \\
\hline C23 & A & 8.07 & 0.003375 & 505000 & 22.44 & 22.67 \\
\hline C23 & $B$ & & & 510000 & 22.67 & \\
\hline $\mathrm{C} 23$ & $\mathrm{C}$ & & & 515000 & 22.89 & \\
\hline $\mathrm{C} 24$ & $A$ & 8.07 & 0.003375 & 457000 & 20.31 & 19.14 \\
\hline $\mathrm{C} 24$ & $B$ & & & 420000 & 18.67 & \\
\hline $\mathrm{C} 24$ & $\mathrm{C}$ & & & 415000 & 18.44 & \\
\hline C25 & A & 8.23 & 0.003375 & 340000 & 15.11 & 19.04 \\
\hline C25 & $B$ & & & 495000 & 22.00 & \\
\hline C25 & $\mathrm{C}$ & & & 450000 & 20.00 & \\
\hline C34 & $A$ & 8.33 & 0.003375 & 470000 & 20.89 & 19.73 \\
\hline C34 & $B$ & & & 462000 & 20.53 & \\
\hline C34 & $\mathrm{C}$ & & & 400000 & 17.78 & \\
\hline C35 & A & 8.47 & 0.003375 & 490000 & 21.78 & 21.11 \\
\hline C35 & $B$ & & & 505000 & 22.44 & \\
\hline C35 & C & & & 430000 & 19.11 & \\
\hline C45 & $A$ & 8.23 & 0.003375 & 410000 & 18.22 & 18.30 \\
\hline C45 & $B$ & & & 380000 & 16.89 & \\
\hline C45 & $\mathrm{C}$ & & & 445000 & 19.78 & \\
\hline
\end{tabular}

Table 4 The 28-day compressive strength values for model validation (control) 4. táblázat A model validálásához használt 28 napos nyomószilárdság értékek

$\begin{array}{lllllllllllllll}\beta_{1} & \beta_{2} & \beta_{3} & \beta_{4} & \beta_{5} & \beta_{12} & \beta_{13} & \beta_{14} & \beta_{15} & \beta_{23} & \beta_{24} & \beta_{25} & \beta_{34} & \beta_{35} & \beta_{45}\end{array}$

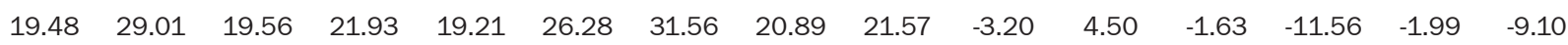

Table 5. Coefficients of Scheffe's second degree polynomial for the regression model

5. táblázat Scheffe másodfokú polinomjának együtthatói a regressziós modellhez 


\section{Regression model for the compressive strength of OSP-cement concrete}

The results of 28-day compressive strength for model formulation of OSP-Cement concrete are a given in Table 3.

Similarly, the results of 28-day compressive strength for model validation of OSP-cement concrete are a given in Table 4.

From Eq. (7), the coefficients of the Scheffe's second degree polynomial are given in Table 5.

Substituting the values of these coefficients into Eq. (6) yields $\widehat{Y}=19.48 \mathrm{X}_{1}+29.01 \mathrm{X}_{2}+19.56 \mathrm{X}_{3}+21.93 \mathrm{X}_{4}+19.21 \mathrm{X}_{5}+$ $26.28 \mathrm{X}_{1} \mathrm{X}_{2}+31.56 \mathrm{X}_{1} \mathrm{X}_{3}+20.89 \mathrm{X}_{1} \mathrm{X}_{4}+21.57 \mathrm{X}_{1} \mathrm{X}_{5}-3.20 \mathrm{X}_{2} \mathrm{X}_{3}+$ $4.50 \mathrm{X}_{2} \mathrm{X}_{4}-1.63 \mathrm{X}_{2} \mathrm{X}_{5}-11.56 \mathrm{X}_{3} \mathrm{X}_{4}-1.99 \mathrm{X}_{3} \mathrm{X}_{5}-9.10 \mathrm{X}_{4} \mathrm{X}_{5}$

Eq. (10) is the regression model for the compressive strength of oyster shell powder-cement concrete using the Scheffe's simplex lattice theory.

\section{Model Validations}

\subsection{Replication Variance}

Mean responses, $Y$ and the variances of replicates, $S_{i}^{2}$ in Table 9 were obtained from Eq. (11).

$Y=\frac{\sum_{i=1}^{n} Y_{i}}{n}$

$S_{i}^{2}=\left\lfloor\frac{1}{n-1}\right\rfloor\left[\sum Y_{i}^{2}\left[\frac{1}{n\left(\sum Y_{i}\right)^{2}}\right]\right]$

Where: $1 \leq \mathrm{i} \leq \mathrm{n}$

Expansion of Eq. (12) gives Eq. (13)

$S_{i}^{2}=\left\lfloor\frac{1}{n-1}\right\rfloor\left[\sum_{i=n}^{n}\left[Y_{i}-Y\right]^{2}\right]$

Where $Y_{i}=$ responses; $Y=$ mean of the responses for each control point; $n=$ number of parallel observations at every point; $n-1=$ degree of freedom; $S i^{2}=$ variance at each design point. For all the design points, $N$, the degree of freedom, $V_{e}$ is given by

$\mathrm{V}_{\mathrm{e}}=\sum N-2=30-2=28$

Where:

$N$ is the number of points.

$S_{y}^{2}=103.24 / 28=3.687$

Where $S_{i}^{2}$ is the variance at each point

$\mathrm{S}_{\mathrm{y}}=1.92$

The results of the compressive strength obtained for the formulation and validation of the model based on Scheffe's lattice theory are given in Table 7.

\subsection{Test of adequacy of the model}

The test for adequacy of the model was done using Student's t-test at 95\% confidence level on the compressive strength at the control points subject to these two hypotheses.

\section{Null hypothesis}

There is no significant difference between the laboratory tests and model predicted strength results.

\section{Alternative hypothesis}

There is a significant difference between the laboratory test and model predicted strength results.

\subsubsection{Student's t-test}

Table 8 shows the parameters with which the student's t-test will be done. We did a two-tailed test (inequality) and if $\mathrm{t}_{\text {Stat }}>\mathrm{t}_{\text {Critical }}$ two-tailed, we reject the null hypothesis.

$$
\mathrm{t}_{\text {Stat }}=\frac{\sum(\mathbf{l a b}-\text { model })}{\sqrt{\frac{\left(15 * \sum\left((\mathbf{l a b}-\text { model })^{2}\right)-\left(\sum(\mathbf{l a b}-\text { model })\right)^{\wedge} 2\right.}{(15-1)}}}=
$$

$\alpha=0.05$ and 0.025 for two tail; DF $=15-1=14$ ( $\mathrm{t}$-distribution table).

$\mathrm{t}_{\text {Critical }}=2.145$

$\mathrm{t}_{\text {Stat }}<\mathrm{t}_{\text {Critical }}$

From the calculations, $\mathrm{t}_{\text {Stat }}=1.868$ and $\mathrm{t}_{\text {Critical }}$ two-tailed $=2.145$, so $t_{\text {Critical }}>t_{\text {Stat }}$. Therefore, we accept the null hypothesis.

\section{Conclusions}

The present study was geared towards the formulation and validation of model to predict the compressive strength of OSPcement concrete, given the mix proportions and vice versa, based on Scheffe's simplex lattice theory. From the foregoing results, the following conclusions are hereby drawn:

i. The statistical tests conducted to validate the Scheffe's lattice model formulated for OSP-cement blended concrete for 28-day compressive strength was found to be adequate for the model.

ii. A good agreement was found between the predicted and experimental values of the 28-day compressive strength, leading to the adoption of null hypothesis.

iii. The model gave highest compressive strength of $30.81 \mathrm{~N} / \mathrm{mm}^{2}$ corresponding to mix ratio of 0.54:0.815:2.045:3.925:0.185 for water, cement, sand, granite and OSP respectively and the minimum compressive strength of $17.85 \mathrm{~N} / \mathrm{mm}^{2}$ corresponding to mix ratio of 0.525:0.825:2.2:4.05:0.175 for water, cement, sand, granite and OSP respectively.

iv. Using the model, compressive strength of all points in the simplex can be derived.

\section{Funding}

This research did not receive any specific grant from funding agencies in the public, commercial, or not-for-profit sectors. 


\begin{tabular}{|c|c|c|c|c|c|c|}
\hline Response & Replicate & Response $Y_{i}\left(\mathrm{~N} / \mathrm{mm}^{2}\right)$ & $\Sigma Y_{i}$ & $\mathbf{Y}$ & $\Sigma Y_{i}^{2}$ & $S_{i}^{2}$ \\
\hline Y1 & A & 17.78 & 58.44 & 19.48 & 1147.11 & 4.26 \\
\hline Y1 & B & 21.78 & & & & \\
\hline $\mathrm{Y} 1$ & C & 18.89 & & & & \\
\hline $\mathrm{Y} 2$ & A & 26.22 & 87.02 & 29.01 & 2544.28 & 10.00 \\
\hline Y2 & B & 32.44 & & & & \\
\hline Y2 & C & 28.36 & & & & \\
\hline Y3 & A & 20.00 & 58.67 & 19.56 & 1147.65 & 0.20 \\
\hline Y3 & B & 19.11 & & & & \\
\hline Y3 & C & 19.56 & & & & \\
\hline Y4 & A & 22.22 & 65.78 & 21.93 & 1445.93 & 1.84 \\
\hline Y4 & B & 20.44 & & & & \\
\hline Y4 & C & 23.11 & & & & \\
\hline Y5 & A & 17.78 & 57.64 & 19.21 & 1115.16 & 3.77 \\
\hline Y5 & B & 21.42 & & & & \\
\hline Y5 & C & 18.44 & & & & \\
\hline $\mathrm{Y} 12$ & A & 28.89 & 92.44 & 30.81 & 2855.11 & 3.23 \\
\hline Y12 & B & 32.44 & & & & \\
\hline Y12 & C & 31.11 & & & & \\
\hline Y13 & A & 31.11 & 82.22 & 27.41 & 2276.54 & 11.52 \\
\hline Y13 & B & 24.44 & & & & \\
\hline $\mathrm{Y} 13$ & C & 26.67 & & & & \\
\hline Y14 & A & 29.78 & 77.78 & 25.93 & 2039.11 & 11.33 \\
\hline Y14 & B & 23.56 & & & & \\
\hline Y14 & C & 24.44 & & & & \\
\hline Y15 & A & 25.78 & 74.22 & 24.74 & 1840.40 & 2.04 \\
\hline Y15 & B & 25.33 & & & & \\
\hline Y15 & C & 23.11 & & & & \\
\hline Y23 & A & 22.44 & 70.44 & 23.48 & 1655.85 & 0.86 \\
\hline Y23 & B & 23.78 & & & & \\
\hline Y23 & C & 24.22 & & & & \\
\hline Y24 & A & 27.33 & 79.78 & 26.59 & 2122.42 & 0.46 \\
\hline Y24 & B & 26.00 & & & & \\
\hline Y24 & C & 26.44 & & & & \\
\hline Y25 & A & 24.00 & 71.11 & 23.70 & 1687.31 & 0.86 \\
\hline Y25 & B & 22.67 & & & & \\
\hline Y25 & C & 24.44 & & & & \\
\hline Y34 & A & 20.22 & 53.56 & 17.85 & 964.89 & 4.41 \\
\hline Y34 & B & 17.11 & & & & \\
\hline Y34 & C & 16.22 & & & & \\
\hline Y35 & A & 19.56 & 56.67 & 18.89 & 1071.06 & 0.35 \\
\hline Y35 & B & 18.67 & & & & \\
\hline Y35 & C & 18.44 & & & & \\
\hline Y45 & A & 18.89 & 54.89 & 18.30 & 1004.83 & 0.28 \\
\hline Y45 & B & 17.87 & & & & \\
\hline Y45 & $C$ & 18.13 & & & & \\
\hline
\end{tabular}




\begin{tabular}{|c|c|c|c|c|c|c|}
\hline C1 & A & 21.33 & 65.56 & 21.85 & 1432.94 & 0.21 \\
\hline C1 & B & 22.22 & & & & \\
\hline $\mathrm{C} 1$ & C & 22.00 & & & & \\
\hline $\mathrm{C} 2$ & A & 28.44 & 73.78 & 24.59 & 1836.64 & 11.13 \\
\hline $\mathrm{C} 2$ & B & 22.67 & & & & \\
\hline $\mathrm{C} 2$ & C & 22.67 & & & & \\
\hline C3 & A & 24.00 & 67.78 & 22.59 & 1536.25 & 2.49 \\
\hline C3 & B & 20.89 & & & & \\
\hline $\mathrm{C} 3$ & C & 22.89 & & & & \\
\hline C4 & A & 27.11 & 73.78 & 24.59 & 1823.90 & 4.76 \\
\hline $\mathrm{C} 4$ & B & 23.33 & & & & \\
\hline C4 & C & 23.33 & & & & \\
\hline C5 & A & 24.44 & 73.33 & 24.44 & 1796.15 & 1.78 \\
\hline C5 & B & 25.78 & & & & \\
\hline $\mathrm{C} 5$ & $C$ & 23.11 & & & & \\
\hline C12 & A & 24.00 & 75.78 & 25.26 & 1916.49 & 1.20 \\
\hline C12 & B & 25.78 & & & & \\
\hline C12 & C & 26.00 & & & & \\
\hline C13 & A & 25.56 & 77.78 & 25.93 & 2016.89 & 0.21 \\
\hline C13 & B & 25.78 & & & & \\
\hline C13 & C & 26.44 & & & & \\
\hline C14 & A & 25.33 & 80.00 & 26.67 & 2136.10 & 1.38 \\
\hline C14 & B & 27.56 & & & & \\
\hline C14 & C & 27.11 & & & & \\
\hline C15 & A & 24.89 & 74.36 & 24.79 & 1848.79 & 2.94 \\
\hline C15 & B & 26.44 & & & & \\
\hline C15 & C & 23.02 & & & & \\
\hline $\mathrm{C} 23$ & A & 22.44 & 68.00 & 22.67 & 1541.43 & 0.05 \\
\hline $\mathrm{C} 23$ & B & 22.67 & & & & \\
\hline C23 & $\mathrm{C}$ & 22.89 & & & & \\
\hline C24 & A & 20.31 & 57.42 & 19.14 & 1101.18 & 1.04 \\
\hline C24 & B & 18.67 & & & & \\
\hline $\mathrm{C} 24$ & C & 18.44 & & & & \\
\hline $\mathrm{C} 25$ & A & 15.11 & 57.11 & 19.04 & 1112.35 & 12.56 \\
\hline $\mathrm{C} 25$ & B & 22.00 & & & & \\
\hline C25 & C & 20.00 & & & & \\
\hline C34 & A & 20.89 & 59.20 & 19.73 & 1174.01 & 2.90 \\
\hline C34 & B & 20.53 & & & & \\
\hline C34 & $C$ & 17.78 & & & & \\
\hline C35 & A & 21.78 & 63.33 & 21.11 & 1343.26 & 3.11 \\
\hline C35 & B & 22.44 & & & & \\
\hline C35 & $\mathrm{C}$ & 19.11 & & & & \\
\hline C45 & A & 18.22 & 54.89 & 18.30 & 1008.44 & 2.09 \\
\hline C45 & B & 16.89 & & & & \\
\hline C45 & C & 19.78 & & & & \\
\hline
\end{tabular}




\begin{tabular}{|c|c|c|}
\hline Symbol & Experimental test & Scheffe's model \\
\hline Y1 & 19.48 & 19.48 \\
\hline Y2 & 29.01 & 29.01 \\
\hline Y3 & 19.56 & 19.56 \\
\hline Y4 & 21.93 & 21.93 \\
\hline Y5 & 19.21 & 19.21 \\
\hline Y12 & 30.81 & 30.81 \\
\hline Y13 & 27.41 & 27.41 \\
\hline Y14 & 25.93 & 25.93 \\
\hline Y15 & 24.74 & 24.74 \\
\hline Y23 & 23.48 & 23.48 \\
\hline Y24 & 26.59 & 26.59 \\
\hline Y25 & 23.70 & 23.70 \\
\hline Y34 & 17.85 & 17.85 \\
\hline Y35 & 18.89 & 18.89 \\
\hline Y45 & 18.30 & 18.30 \\
\hline C1 & 21.85 & 23.14 \\
\hline C2 & 24.59 & 23.01 \\
\hline C3 & 22.59 & 21.36 \\
\hline C4 & 24.59 & 22.44 \\
\hline C5 & 24.44 & 17.97 \\
\hline C12 & 25.26 & 21.45 \\
\hline C13 & 25.93 & 24.20 \\
\hline C14 & 26.67 & 24.14 \\
\hline C15 & 24.79 & 22.56 \\
\hline $\mathrm{C} 23$ & 22.67 & 23.50 \\
\hline $\mathrm{C24}$ & 19.14 & 18.60 \\
\hline $\mathrm{C25}$ & 19.04 & 20.23 \\
\hline C34 & 19.73 & 18.95 \\
\hline C35 & 21.11 & 21.39 \\
\hline C45 & 18.30 & 21.14 \\
\hline
\end{tabular}

References

[1] Oguaghamba, O. A. - Onyia, M. E.: Modified and generalized full cubic polynomial response surface methodology in engineering mixture design, Nigerian Journal of Engineering and Technology, 2019; 38 (1), 52-59

[2] Mlinarik, Lilla - Kopecsko, Katalin - Borosnyoi, Adorjan: Properties of cement mortars in fresh and hardened condition influenced by combined application of SCMs, Epitosanyag - Journal of Silicate Based and Composite Materials, Vol. 68, No. 2 (2016), 62-66 p. http://dx.doi.org/10.14382/epitoanyag-jsbcm.2016.11

[3] Scheffe, H.: Experiments with mixtures, Journal of Royal Statistical Society, 1958; Series B, 1958; 20: 344-366

[4] Scheffe H. : Simplex-centroid design for experiments with mixtures, Journal of Journal of the Royal Statistical Society", Series B, 1963; 25: 235-263

[5] House R. : Simplex lattice designs, 2017; retrieved from http://reliawiki.org/index.php/Mixture_Design on $10^{\text {th }}$ August, 2017.

[6] Oguaghamba, O. A. - Mama, B. O.: Generalized Scheffe’s second degree mathematical methods approach in engineering mixture design", Proceedings of $16^{\text {th }}$ International Conference and AGM of Nigerian Institution of Civil Engineers (NICE) on Transforming national economy through sustainable civil engineering infrastructures- Engineering solutions to problematic soils and allied construction materials, Calabar, Nigeria, Oct. 24-26, 2018, 32-44

[7] Brown, L. J.: General blending models for mixture experiments: Design and analysis", An unpublished Ph.D thesis submitted to Faculty of Engineering and Physical Sciences, University of Manchester, United Kingdom, 2014

[8] Etuk, B. R. - Etuk, I. F. - Asuquo, L. O.:The feasibility of using sea shell ash admixtures for concrete, Journal of Environmental Sciences and Engineering, 2012; A1: 121-127

[9] Lerwattanaruk, P. - Makul, N. - Siripattarapravat, C.: Utilization of ground waste sea shells in cement mortars for masonry and plastering, Journal of Environmental Sciences and Management, 2012; 111: 133-141

[10] Liang Chou-Fu, - Wang Hung-Yu, : Feasibility of pulverized oyster shell as a cementing material, Advances in Materials Sciences and Engineering, Hindawi Publishing Corporation, 2013

[11] Wen-Ten, Kuo - Her-Yung, Wang - Chun-Ya, Shu - De-Sin, Su: Engineering properties of controlled low-strength material containing waste oyster shells, Construction and Building Materials, 2013; 46, 128-133

[12] Zhong, Bin-Yang - Zho, Qiang-Chan - Chan, Chang-Feng - Yan, Yu: Structure and property characterization of oyster shell cementing material, Chinese Journal of Struct. Chem., 2012; 31 (1), 85-92

[13] Ubachukwu, O. A. - Okafor, F. O.: Investigation of the supplementary cementitious potentials of oyster shell powder for eco-friendly and lowcost concrete, Electronic Journal of Geotechnical Engineering (EJGE), 2019, 24 (05), pp. 1297-1306.

Table 7 Experimental test and Scheffe's model results

7. táblázat Kisérleti és Scheffe modell eredmények

\begin{tabular}{lcccc} 
Symbol & Lab & Model & Lab-Model & (Lab-Model) ${ }^{\wedge} \mathbf{2}$ \\
$\mathbf{C 1}$ & 21.85 & 23.14 & -1.29 & 1.67 \\
\hline $\mathbf{C 2}$ & 24.59 & 23.01 & 1.58 & 2.50 \\
\hline $\mathbf{C 3}$ & 22.59 & 21.36 & 1.23 & 1.52 \\
\hline $\mathbf{C 4}$ & 24.59 & 22.44 & 2.15 & 4.61 \\
\hline $\mathbf{C 5}$ & 24.44 & 17.97 & 6.48 & 41.94 \\
\hline $\mathbf{C 1 2}$ & 25.26 & 21.45 & 3.81 & 14.51 \\
\hline $\mathbf{C 1 3}$ & 25.93 & 24.20 & 1.73 & 2.98 \\
\hline $\mathbf{C 1 4}$ & 26.67 & 24.14 & 2.53 & 6.39 \\
\hline $\mathbf{C 1 5}$ & 24.79 & 22.56 & 2.23 & 4.96 \\
\hline $\mathbf{C 2 3}$ & 22.67 & 23.50 & -0.83 & 0.70 \\
\hline $\mathbf{C 2 4}$ & 19.14 & 18.60 & 0.54 & 0.29 \\
\hline $\mathbf{C 2 5}$ & 19.04 & 20.23 & -1.20 & 1.43 \\
\hline $\mathbf{C 3 4}$ & 19.73 & 18.95 & 0.78 & 0.61 \\
\hline $\mathbf{C 3 5}$ & 21.11 & 21.39 & -0.27 & 0.08 \\
\hline $\mathbf{C 4 5}$ & 18.30 & 21.14 & -2.84 & 8.06 \\
\hline & Total & & $\mathbf{1 6 . 6 1}$ & $\mathbf{9 2 . 2 4}$
\end{tabular}

[14] Nehme, Salem G.: Influence of supplementary cementing materials on conventional and self-compacting concretes, Epitoanyag - Journal of Silicate Based and Composite Materials, Vol. 67, No. 1 (2015), 28-33 http://dx.doi.org/10.14382/epitoanyag-jsbcm.2015.6

[15] BS12: Specification for Portland cement, British Standards Institution, London, 1996

[16] BS 812: Part 1: Method of determination of particle size and shape, British Standards Institution, London, 1975

[17] BS882: Specification for aggregates from natural sources for concrete, British Standards Institution, London, 1992

[18] BS3140: Methods of test for water for making concrete, including notes on the suitability of the water, British Standards Institution, London, 1980

[19] Okafor, F. O. - Oguaghamba, O. A.: Procedure for optimization using Scheffe's models, Journal of Engineering Science and Applications, 2010, 7 (1), 36-47

[20] Osadebe, N. N. - Ibearugbulem, O. M.: Application of Osadebe's alternative regression model in optimizing compressive strength of periwinkle shells-granite concrete, Nigerian Society of Engineers Technical Transaction, 2009, 43(1), 47-59

$\underline{\text { Ref.: }}$

Ubachukwu, Obiekwe A. - Okafor, Fidelis O.: Formulation of predictive model for the compressive strength of oyster shell powdercement concrete using Scheffe's simplex lattice theory Építőanyag - Journal of Silicate Based and Composite Materials, Vol. 72, No. 6 (2020), 210-218. p.

https://doi.org/10.14382/epitoanyag-jsbcm.2020.34 